\title{
Impact of major different variants of papillary thyroid microcarcinoma on the clinicopathological characteristics: the study of 1041 cases
}

\author{
Jingtai Zhi ${ }^{1} \cdot$ Jingzhu Zhao $^{1} \cdot$ Ming Gao $^{1} \cdot \mathrm{Yi} \mathrm{Pan}^{2} \cdot \mathrm{Jianghua} \mathrm{Wu}^{2} \cdot \mathrm{Yigong} \mathrm{Li}^{1} \cdot$ \\ Dapeng $\mathrm{Li}^{1} \cdot$ Yang $\mathrm{Yu}^{1} \cdot$ Xiangqian $\mathrm{Zheng}^{1}$
}

Received: 6 March 2017 / Accepted: 19 July 2017 / Published online: 25 July 2017

(C) The Author(s) 2017. This article is an open access publication

\begin{abstract}
Background The incidence of papillary thyroid microcarcinoma (PTMC) has been increasing globally in the past few decades. PTMC does not have a distinctive morphology that results in differences in biological behavior. The aim of this study was to classify PTMCs according to the morphological features and explore the relationship with clinicopathological characteristics. Additionally, we sought to evaluate whether different variants of PTMC can be an independent predictor for lymph mode metastasis when considering other risk factors.

Methods Between December 2014 and December 2015, 1041 PTMC cases undergoing surgical resection at Tianjin Medical University Cancer Institute and Hospital were reviewed retrospectively. Statistical analysis was performed to investigate the independent factors for lymph node metastasis in PTMC.
\end{abstract}

Jingtai Zhi and Jingzhu Zhao contributed equally and should be considered joint first authors.

Ming Gao

headandneck2008@126.com

Xiangqian Zheng

xiangqian_zheng@163.com

1 Department of Thyroid and Neck Tumor, National Clinical Research Center for Cancer, Key Laboratory of Cancer Prevention and Therapy, Tianjin's Clinical Research Center for Cancer, Tianjin Medical University Cancer Institute and Hospital, Tianjin 300060, People's Republic of China

2 Department of Pathology, National Clinical Research Center for Cancer, Key Laboratory of Cancer Prevention and Therapy, Tianjin's Clinical Research Center for Cancer, Tianjin Medical University Cancer Institute and Hospital, Tianjin 300060, People's Republic of China
Results Conventional variant PTMC (CPTMC), follicular variant PTMC (FPTMC), and encapsulated variant PTMC (EnPTMC) were major variants in PTMC, collectively accounting for $96.7 \%$ of the entire PTMC cohort.There were significant differences in clinicopathological characteristics among the three major variants. The frequency of aggressive parameters was significantly different among the three variants, including tumor size, minimal extrathyroidal extension (minimal ETE), and lymph node metastasis (all $P<0.05$ ), being highest in CPTMC, lowest in EnPTMC, and intermediate in FPTMC. FPTMC (OR $=0.642$, $P=0.003)$ and EnPTMC $(\mathrm{OR}=0.540, P=0.041)$ were independent protective factors for lymph node metastasis (LNM). In contrast, male gender $(\mathrm{OR}=1.836, P=0.000)$, age less than 45 years $(\mathrm{OR}=1.457, P=0.009)$, tumor size greater than $0.5 \mathrm{~cm}(\mathrm{OR}=1.453, P=0.007)$, calcification $(\mathrm{OR}=1.465, P=0.016)$, minimal ETE $(\mathrm{OR}=1.801$, $P=0.001)$, and multifocality $(\mathrm{OR}=1.721, P=0.000)$ were independent risk factors for LNM.

Conclusions The present study demonstrates the distinct biological behaviors of the three major PTMC variants and establishes an aggressive order of CPTMC $\gg$ FPTMC $>$ EnPTMC. It is necessary to take into consideration variant-related risks and other independent predictors for the determination of lymphadenectomy in patients with PTMC.

Keywords Papillary thyroid microcarcinoma - Variant . Clinicopathological characteristics
Abbreviations
PTMC
PTC
Papillary thyroid microcarcinoma
FNAB Papillary thyroid carcinoma
LNM Fine-needle aspiration biopsy Lymph node metastasis 


$\begin{array}{ll}\text { Minimal ETE } & \text { Minimal extrathyroidal extension } \\ \text { HT } & \text { Hashimoto's thyroiditis } \\ \text { CPTMC } & \text { Conventional variant PTMC } \\ \text { FPTMC } & \text { Follicular variant PTMC } \\ \text { EnPTMC } & \text { Encapsulated variant PTMC } \\ \text { E-FPTC } & \text { Encapsulated follicular variant of PTC } \\ \text { E-CPTC } & \text { Encapsulated conventional variant of PTC }\end{array}$

\section{Introduction}

Papillary thyroid microcarcinoma (PTMC), defined as papillary thyroid carcinoma (PTC) $10 \mathrm{~mm}$ or less in diameter, has been dramatically rising in incidence during the past few decades, accounting for nearly half the increase in PTC [1-4]. PTMC is generally regarded as an indolent disease but does pose a risk for local recurrence and distant metastasis [5, 6]. Identification of aggressive cases from favorable ones is necessary for the patient-tailored treatment of PTMC.

PTMC does not have a distinctive morphology, which means that all cellular features and growth patterns that can be found in other variants of PTC can be observed in PTMC [7, 8]. In PTC, the variant-related differences in biological behavior and prognosis are very large. Compared with the conventional variant, solid variant, diffuse sclerosing variant, and tall cell variant are recommended as the aggressive types, and follicular variant and the Warthin-like variant are considered favorable [9-14], which has further led to variant-related changes in PTC treatment $[11,13,15,16]$. Considering the findings of these studies, the appearance of other variants in PTMC may incur variant-relevant risk, which has a profound impact on the treatment strategies. The purpose of this study was to classify the PTMCs according to the nature of the tumor boundaries, distinct architecture, or cellular characteristics and explore the clinicopathological characteristics of different variants of PTMC. Additionally, we sought to evaluate whether different variants of PTMC can be an independent predictor for lymph mode metastasis when considering other risk factors.

\section{Materials and methods}

This retrospective cohort review research was supported by the academic ethics board, and informed consent was obtained from all patients to allow their information to be used for the study.

\section{Patients}

We consecutively collected 1041 patients from all the PTMC cases between December 2014 and December 2015 in our hospital. Exclusion criteria were (1) patients had other malignancy, (2) no lymph node was resected, (3) distant metastasis, (4) patients with a history of previous operation for PTC, (5) largest tumor greater than $1 \mathrm{~cm}$, and (6) age less than 18 years. All cases were grouped according to the subtype of the largest tumor. These chosen patients were mostly asymptomatic, and their lesions were accidently discovered during the annual physical examination. Once the nodules were suspected for malignancy by fine-needle aspiration biopsy (FNAB) or ultrasound (US), either thyroid lobectomy or total/near total thyroidectomy combined with prophylactic central neck dissection was performed in all patients. Patients who had preoperatively or intraoperatively proven lateral neck metastasis by FNAB or biopsy underwent therapeutic lateral neck dissection.

\section{Pathological examination}

Both intraoperative frozen and postoperative paraffin sections were submitted to two experienced endocrine pathologists (Yi Pan and Jianghua Wu) to establish the diagnosis of PTMC and other characteristic pathological parameters, including histopathological types, multifocality, coexistence of Hashimoto's thyroiditis (HT), minimal extrathyroidal extension (minimal ETE), status of lymph node metastasis (LNM), and tumor size, fibrosis, and calcification of the largest tumor. All the pathological parameters were identified according to Rosai and Ackerman's surgical pathology, 10th edition. Representative sections were examined in a blinded fashion, and the concordance rate was $97 \%$. The final diagnoses of discordant or rare-variant cases were based on the consensus of two pathologists.

\section{Statistical analysis}

Differences among variants were compared by the chisquare test. Univariate and multivariate logistic regression models were used to evaluate independent predictors for lymph mode metastasis in PTMC. $P<0.05$ was assumed as statistical significance. SPSS version 22.0.0 (IBM, Armonk, NY, USA) was used to perform the statistical analysis.

\section{Results}

There were 1041 PTMC patients enrolled in this study. The major variants of PTMC, including conventional 

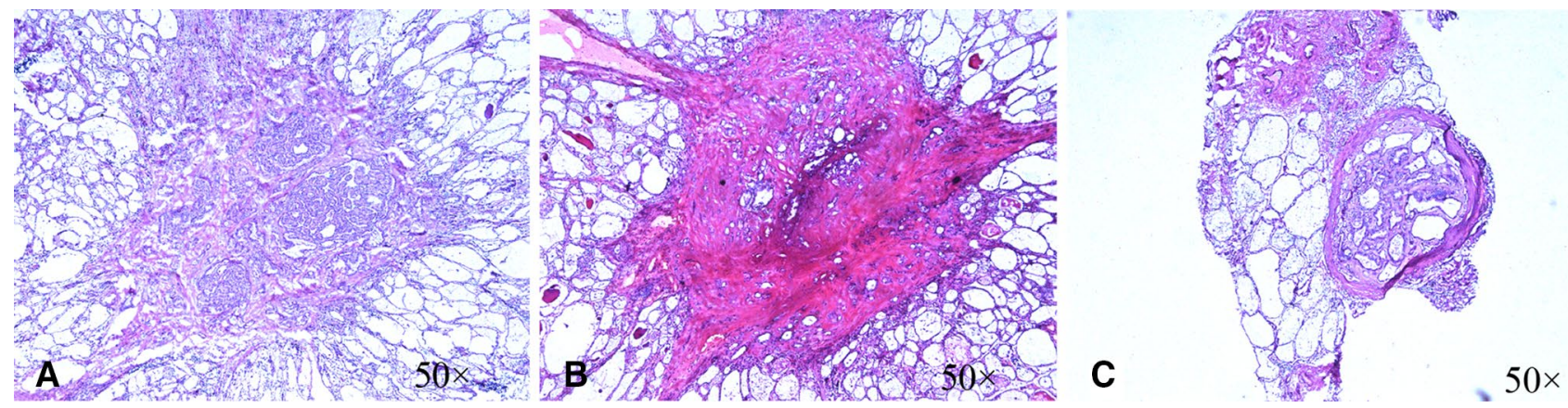

Fig. 1 a Conventional variant: characterized by papillary architecture, $\Phi=2 \mathrm{~mm}$. b Follicular variant: a papillary carcinoma composed entirely or almost entirely of follicles, $\Phi=3 \mathrm{~mm}$. c Encapsulated variant: a papillary carcinoma totally surrounded by a capsule, $\Phi=1 \mathrm{~mm}$

Table 1 Distribution of variants among 1041 patients between December 2014 and December 2015

\begin{tabular}{lrr}
\hline Subtype & $N$ & Present \\
\hline Conventional & 471 & 45.2 \\
Follicular & 454 & 43.6 \\
Encapsulated & 82 & 7.9 \\
Warthin-like & 17 & 1.6 \\
Solid & 3 & 0.3 \\
Oncocytic & 3 & 0.3 \\
Tall cell & 6 & 0.6 \\
Trabecular & 5 & 0.5 \\
\hline
\end{tabular}

variant (CPTMC, 471 cases, $45.2 \%$; Fig. 1a), follicular variant (FPTMC, 454 cases, 43.6\%; Fig. 1b), and encapsulated variant (EnPTMC, 82 cases, 7.9\%; Fig. 1c), collectively accounted for $96.7 \%$ of the entire PTMC cohort. Other variants of PTMC (e.g., Warthin-like variant, solid variant, oncocytic variant, tall cell variant, or trabecular variant) were rare (collectively, 34 cases, $3.3 \%$ ), so we only analyzed the clinicopathological characteristics of the 1007 cases with the major variants. The sex ratio was 1:3.86 (male:female, 207:800). The age range was 18-73 (mean, $46 \pm 9.5$ ) years. The size of the largest tumor was $5.7 \pm 1.8$ (mean $\pm \mathrm{SD}$ ) $\mathrm{mm}$. The rate of fibrosis and calcification was $98.5 \%$ and $31.7 \%$, respectively. Multifocal tumors were found in 364 cases (Table 1). Minimal ETE was seen in $200(19.9 \%)$ patients, including 59 in vascular, 120 in fat, 8 in striated muscle, 1 in nerve, and 12 multiple minimal ETE. HT was present in $26.3 \%$ of the patients. The frequency of LNM was 26.3\%; among these, 275 were N1a and 40 were N1b (Table 2). Most patients (94.6\%) underwent prophylactic central neck dissection; in only 54 patients was central + lateral neck dissection performed.

\section{Comparison of the clinicopathological data among three variants of PTMC}

Comparisons of the clinicopathological features of the three PTMC variants are summarized in Table 3. Age, calcification, MEE, tumor size, and LNM showed significant differences among three variant-specific groups. The aggressive parameters, including MEE, tumor size, and lymph node metastasis, were highest in CPTMC, lowest in EnPTMC, and generally intermediate in FPTMC. In contrast with this order, the prevalence of age of 45 years or more and calcification was highest in EnPTMC, lowest in CPTMC, and generally intermediate in FPTMC. These patterns were further confirmed on pair-wise comparison (Table 4). Specifically, the prevalence of patient age of 45 years or more was similar between FPTMC and EnPTMC and significantly less common in CPTMC. The rate of calcification was similar between CPTMC and FPTMC and significantly higher in EnPTMC. Tumor size was similar between FPTMC and EnPTMC, and both were smaller than CPTMC. The incidence of minimal ETE showed no difference between CPTMC and FPTMC, but minimal ETE was significantly less commonly seen in EnPTMC than in CPTMC and FPTMC. The occurrence of LNM was significantly higher in CPTMC than in FPTMC and EnPTMC.

\section{Correlation between clinicopathological features and LNM}

Univariate logistic regression analysis indicated that gender, age, subtypes, MEE, multifocality, and tumor size were all strongly associated with the LNM in PTMC $(P<0.05)$. There was a significant difference in the occurrence of LNM between CPTMC with calcification and without calcification. In the other two subtypes, however, calcification was not remarkably correlated with incidence of LNM. Multiple logistic regression analysis indicated that male gender (OR, 1.896; 95\% CI, 1.355-2.651), age45 years or less (OR, 1.393; 95\% CI, 1.053-1.842), tumor size (OR, 1.453; 95\% CI, 1.068-1.977), minimal ETE (OR, 1.801; 95\% CI, 1.257-2.579), and multifocality (OR, 1.530; 95\% CI, 1.129-2.074) were all significantly connected 
Table 2 Clinicopathological characteristics of patients with three major variants of papillary thyroid microcarcinoma (PTMC)

\begin{tabular}{|c|c|c|}
\hline Characteristics & $N($ mean $\pm \mathrm{SD})$ & Present \\
\hline \multicolumn{3}{|l|}{ Gender } \\
\hline Female & 800 & 79.4 \\
\hline Male & 207 & 20.6 \\
\hline Age (years) & $46 \pm 9.5$ & \\
\hline$\geq 45$ & 558 & 55.4 \\
\hline$<45$ & 449 & 44.6 \\
\hline Tumor size & $0.57 \pm 0.18$ & \\
\hline$>5 \mathrm{~mm}$ & 510 & 50.6 \\
\hline$\leq 5 \mathrm{~mm}$ & 497 & 49.4 \\
\hline \multicolumn{3}{|l|}{ Fibrosis } \\
\hline Absent & 15 & 1.5 \\
\hline Present & 992 & 98.5 \\
\hline \multicolumn{3}{|l|}{ Calcification } \\
\hline Absent & 688 & 68.3 \\
\hline Present & 319 & 31.7 \\
\hline \multicolumn{3}{|l|}{ Minimal ETE } \\
\hline Absent & 807 & 80.1 \\
\hline Present & 200 & 19.9 \\
\hline Vascular & 59 & 5.9 \\
\hline Fat & 120 & 11.9 \\
\hline Striated muscle & 8 & 0.8 \\
\hline Nerve & 1 & 0.1 \\
\hline Multiple MEE & 12 & 1.2 \\
\hline \multicolumn{3}{|l|}{ Coexistence of HT } \\
\hline Absent & 742 & 73.7 \\
\hline Present & 265 & 26.3 \\
\hline \multicolumn{3}{|l|}{ Multifocality } \\
\hline Absent & 643 & 63.9 \\
\hline Present & 364 & 36.1 \\
\hline \multicolumn{3}{|c|}{ Lymph node metastases } \\
\hline Absent & 692 & 68.7 \\
\hline Present & 315 & 31.3 \\
\hline \multicolumn{3}{|l|}{$\mathrm{N}$-stage } \\
\hline No & 692 & 68.7 \\
\hline N1a & 275 & 27.3 \\
\hline N1b & 40 & 4.0 \\
\hline \multicolumn{3}{|c|}{ Neck excision extent } \\
\hline Central & 953 & 94.6 \\
\hline Central + lateral & 54 & 5.4 \\
\hline
\end{tabular}

Minimal ETE minimal extrathyroidal extension, HT Hashimoto's thyroiditis, $L N M$ lymph node metastases

with the LNM in PTMC $(P<0.05)$. Compared with CPTMC, FPTMC showed significant differences $(P=0.006)$, which means that FPTMC (OR, 0.660; 95\% CI, 0.492-0.885) was an independent protective factor for LNM. Although the $P$ value $>0.05$, the OR of EnPTMC was 0.625 , also indicating the indolent nature of EnPTMC (Table 5).
Table 3 Comparison of the clinicopathological data among three variants of PTMC

\begin{tabular}{|c|c|c|c|c|c|}
\hline $\begin{array}{l}\text { Character- } \\
\text { istic }\end{array}$ & CPTMC & FPTMC & EnPTMC & $\chi^{2}$ & $P$ \\
\hline \multicolumn{6}{|l|}{ Gender } \\
\hline Female & $360(76.4 \%)$ & $373(82.2 \%)$ & $67(81.7 \%)$ & \multirow[t]{2}{*}{4.920} & \multirow[t]{2}{*}{0.085} \\
\hline Male & $111(23.6 \%)$ & $81(17.8 \%)$ & $15(18.3 \%)$ & & \\
\hline \multicolumn{6}{|l|}{ Age (years) } \\
\hline$\geq 45$ & $239(50.7 \%)$ & $263(57.9 \%)$ & $56(68.3 \%)$ & \multirow[t]{2}{*}{10.827} & \multirow[t]{2}{*}{0.004} \\
\hline$<45$ & $232(49.3 \%)$ & $191(42.1 \%)$ & $26(31.7 \%)$ & & \\
\hline \multicolumn{6}{|l|}{ Fibrosis } \\
\hline Absent & $11(2.3 \%)$ & $4(0.9 \%)$ & $0(0.0 \%)$ & \multirow[t]{2}{*}{4.682} & \multirow[t]{2}{*}{0.096} \\
\hline Present & $460(97.7 \%)$ & $450(99.1 \%)$ & $82(100 \%)$ & & \\
\hline \multicolumn{6}{|c|}{ Calcification } \\
\hline Absent & $351(74.5 \%)$ & $308(67.8 \%)$ & $29(35.4 \%)$ & \multirow[t]{2}{*}{49.564} & \multirow[t]{2}{*}{$<0.001$} \\
\hline Present & $120(25.5 \%)$ & $146(32.2 \%)$ & $53(64.6 \%)$ & & \\
\hline \multicolumn{6}{|c|}{ Minimal ETE } \\
\hline Absent & $370(78.6 \%)$ & $355(78.2 \%)$ & $82(100 \%)$ & \multirow[t]{2}{*}{22.143} & \multirow[t]{2}{*}{$<0.001$} \\
\hline Present & $101(21.4 \%)$ & $99(21.8 \%)$ & $0(0.0 \%)$ & & \\
\hline \multicolumn{6}{|c|}{ Coexistence of HT } \\
\hline Absent & $339(72.0 \%)$ & $344(75.8 \%)$ & $59(72.0 \%)$ & \multirow[t]{2}{*}{1.857} & \multirow[t]{2}{*}{0.395} \\
\hline Present & $132(28.0 \%)$ & $110(24.2 \%)$ & $23(28.0 \%)$ & & \\
\hline \multicolumn{6}{|c|}{ Multifocality } \\
\hline Absent & $302(64.1 \%)$ & $285(62.8 \%)$ & $56(68.3 \%)$ & \multirow[t]{2}{*}{0.943} & \multirow[t]{2}{*}{0.624} \\
\hline Present & $169(35.9 \%)$ & $169(37.2 \%)$ & $26(31.7 \%)$ & & \\
\hline \multicolumn{6}{|l|}{ Tumor size } \\
\hline$>5 \mathrm{~mm}$ & $272(57.7 \%)$ & $204(44.9 \%)$ & $34(41.5 \%)$ & \multirow[t]{2}{*}{18.200} & \multirow[t]{2}{*}{$<0.001$} \\
\hline$\leq 5 \mathrm{~mm}$ & $199(42.3 \%)$ & $250(55.1 \%)$ & $48(58.5 \%)$ & & \\
\hline \multicolumn{6}{|l|}{ LNM } \\
\hline Absent & $296(62.8 \%)$ & $332(73.1 \%)$ & $64(78.0 \%)$ & \multirow[t]{2}{*}{14.986} & \multirow[t]{2}{*}{0.001} \\
\hline Present & $175(37.2 \%)$ & $122(26.9 \%)$ & $18(22.0 \%)$ & & \\
\hline
\end{tabular}

CPTMC conventional variant PTMC, FPTMC follicular variant PTMC, EnPTMC encapsulated variant PTMC, minimal ETE minimal extrathyroidal extension, $H T$ Hashimoto's thyroiditis, $L N M$ lymph node metastases

Table 4 Pairwise comparison of clinicopathological features among three variants of PTMC

\begin{tabular}{lccc}
\hline Characteristic & $\begin{array}{l}\text { CPTMC versus } \\
\text { FPTMC }\end{array}$ & $\begin{array}{l}\text { FPTMC versus } \\
\text { EnPTMC }\end{array}$ & $\begin{array}{l}\text { EnPTMC } \\
\text { versus } \\
\text { CPTMC }\end{array}$ \\
\hline Age & 0.028 & 0.078 & 0.003 \\
Calcification & 0.025 & $<0.001$ & $<0.001$ \\
Minimal ETE & 0.894 & $<0.001$ & $<0.001$ \\
Tumor size & $<0.001$ & 0.560 & 0.006 \\
LNM & 0.001 & 0.351 & 0.008
\end{tabular}

CPTMC conventional variant PTMC, FPTMC follicular variant PTMC, EnPTMC encapsulated variant PTMC, minimal ETE minimal extra-thyroidal extension, $H T$ Hashimoto's thyroiditis, $L N M$ lymph node metastases 
Table 5 Univariate analysis of lymph node metastasis and clinicopathological features in PTMC

\begin{tabular}{|c|c|c|c|c|}
\hline Characteristic & LNM- & LNM+ & $\chi^{2}$ & $P$ \\
\hline \multicolumn{5}{|l|}{ Gender } \\
\hline Female & 573 & 227 & 15.289 & $<0.001$ \\
\hline Male & 119 & 88 & & \\
\hline \multicolumn{5}{|l|}{ Age (years) } \\
\hline$\geq 45$ & 406 & 152 & 9.506 & 0.002 \\
\hline$<45$ & 286 & 163 & & \\
\hline \multicolumn{5}{|l|}{ Subtypes } \\
\hline СРТМС & 296 & 175 & 14.986 & 0.001 \\
\hline FPTMC & 332 & 122 & & \\
\hline EnPTMC & 64 & 18 & & \\
\hline \multicolumn{5}{|l|}{ Fibrosis } \\
\hline Absent & 9 & 6 & 0.538 & 0.463 \\
\hline Present & 683 & 309 & & \\
\hline \multicolumn{5}{|l|}{ Calcification } \\
\hline \multicolumn{5}{|l|}{ СРТМС } \\
\hline Absent & 232 & 119 & 6.239 & 0.012 \\
\hline Present & 64 & 56 & & \\
\hline \multicolumn{5}{|l|}{ FPTMC } \\
\hline Absent & 232 & 76 & 2.352 & 0.125 \\
\hline Present & 100 & 46 & & \\
\hline \multicolumn{5}{|l|}{ EnPTMC } \\
\hline Absent & 24 & 5 & 0.581 & 0.446 \\
\hline Present & 40 & 13 & & \\
\hline \multicolumn{5}{|l|}{ Minimal ETE } \\
\hline Absent & 585 & 222 & 26.890 & 0.000 \\
\hline Present & 107 & 93 & & \\
\hline \multicolumn{5}{|c|}{ Coexistence of HT } \\
\hline Absent & 505 & 237 & 0.571 & 0.450 \\
\hline Present & 187 & 78 & & \\
\hline \multicolumn{5}{|l|}{ Multifocality } \\
\hline Absent & 474 & 169 & 20.671 & 0.000 \\
\hline Present & 218 & 146 & & \\
\hline \multicolumn{5}{|l|}{ Tumor size } \\
\hline$>5 \mathrm{~mm}$ & 311 & 199 & 28.788 & 0.000 \\
\hline$\leq 5 \mathrm{~mm}$ & 381 & 116 & & \\
\hline
\end{tabular}

CPTMC conventional variant PTMC, FPTMC follicular variant PTMC, EnPTMC encapsulated variant PTMC, minimal ETE minimal extrathyroidal extension, HT Hashimoto's thyroiditis, LNM lymph node metastases

\section{Discussion}

The American Thyroid Association (ATA) guidelines recommend thyroidectomy without prophylactic central neck dissection is appropriate for small (T1 or T2), noninvasive, clinically node-negative (cN0) PTC [15]. In cases whose largest tumor is $>10 \mathrm{~mm}$, this practice successfully keeps the balance of the risk caused by the enlarged surgery extent and the benefit of reduction in recurrence. In PTMC, however, the balance is remarkably disturbed by variantspecific differences without sufficient attention. Although PTMC is a subset of PTC, variants of PTMC differ from those of PTC in two respects: clinicopathological features and variant distribution, resulting in an unbalanced extent of surgery in PTMC patients (Table 6).

Encapsulated variant is defined as a papillary carcinoma totally surrounded by a capsule. In the present study, EnPTMC showed excellent biological behavior, being the lowest in aggressive parameters (tumor size and incidence of minimal ETE and LNM) among three variants. Because several authors have reported that age less than 45 years had a higher incidence of lymph node metastasis $[17,18]$, the age distribution also indicated the indolent nature of EnPTMC. The previous studies of PTC grouped the encapsulated variant according to the variant inside, underlining the benign behavior of the encapsulated follicular variant of PTC (E-FPTC) [19, 20]. One study reported that E-FPTC resembled the adenoma/follicular carcinoma group of tumors in its capsular/vascular invasive pattern and its propensity for lymph node metastasis whereas E-CPTC behaved more like conventional PTC [21]. In 2016, E-FPTC was categorized as low risk by the ATA [15]. In the present study, however, there was no significant difference in any respect between the encapsulated conventional variant of PTMC (E-CPTMC) and the encapsulated follicular variant of PTMC (not shown in the table), and both were shown as indolent. Overtreatment may occur in patients with EnPTMC (especially E-CPTMC), which needs further studies to determine the optimal extent of surgery.

Follicular variant is defined as a papillary carcinoma composed entirely or almost entirely of follicles. FPTMC was entirely intermediate in all parameters between CPTMC and EnPTMC. Except for the incidence of minimal ETE, all the aggressive parameters including age, tumor size distribution, and the occurrence rate of LNM in FPTMC was analogous to that of EnPTMC, remarkably differentiated from that of CPTMC. These findings are supported by the previous studies on PTC [9, 22]. In the present study, FPTMC accounted for $43.6 \%$ of the 1041 PTMCs, whose prevalence was nearly equal to that of CPTMC. This finding was particularly worth noting because several reports mentioned that the incidence of CPTC was 2 to 4.5 times higher than that of FPTC [9, 23, 24]. One reasonable hypothesis for the decrease in proportion of follicular variant from PTMC to PTC is that, compared with CPTMCs, fewer FPTMCs enlarge in tumor size and develop into FPTC. In spite of the absence of relevant evidence, this hypothesis may have some association with the phenomenon that quite a number of PTMCs enlarged slowly or almost did not enlarge [25, 26]. In our study, the size of tumor was significantly smaller in FPTMCs than 
Table 6 Multivariate analysis of lymph node metastasis and clinicopathological features in PTMC

\begin{tabular}{lrlrllll}
\hline Characteristics & \multicolumn{1}{l}{$B$} & SE & Wald & $P$ & OR & \multicolumn{2}{c}{$95 \%$ CI } \\
\cline { 5 - 8 } & & & & & & Lower & Upper \\
\hline Gender & 0.640 & 0.171 & 13.964 & 0.000 & 1.896 & 1.355 & 2.651 \\
Age & -0.331 & 0.143 & 5.381 & 0.020 & 1.393 & 1.053 & 1.842 \\
Subtypes & & & & & & & \\
CPTMC & & & 8.681 & 0.013 & Reference & & \\
FPTMC & -0.415 & 0.150 & 7.687 & 0.006 & 0.660 & 0.492 & 0.885 \\
EnPTMC & -0.469 & 0.296 & 2.522 & 0.112 & 0.625 & 0.350 & 1.116 \\
Tumor size & 0.426 & 0.155 & 7.527 & 0.06 & 1.530 & 1.129 & 2.074 \\
Fibrosis & -0.327 & 0.549 & 0.356 & 0.551 & 0.721 & 0.246 & 2.112 \\
Minimal ETE & 0.581 & 0.182 & 10.153 & 0.001 & 1.788 & 1.251 & 2.557 \\
Coexistence of HT & -0.060 & 0.166 & 0.130 & 0.719 & 0.942 & 0.680 & 1.305 \\
Multifocality & 0.537 & 0.147 & 13.282 & 0.000 & 1.712 & 1.282 & 2.285 \\
Constant & -1.400 & 0.644 & 4.725 & 0.030 & 0.247 & & \\
\hline
\end{tabular}

CPTMC conventional variant PTMC, FPTMC follicular variant PTMC, EnPTMC encapsulated variant PTMC, minimal ETE minimal extrathyroidal extension, $H T$ Hashimoto's thyroiditis in CPTMCs $(44.9 \%$ vs. $55.7 \%, P<0.001)$. The change in variant distribution indicates the specific growth pattern on FPTMC, deserving further studies and cautious selection in surgery extent.

In our univariate and multivariate analysis, LNM was significantly associated with male gender, age less than 45 years, tumor size, minimal ETE, and multifocality, which was supported by the recent study [6, 27, 28]. Compared with CPTMC, FPTMC was an independent protective factor for LNM, again demonstrating the indolent nature of this variant.

Calcification has been reported as a significant indicator for nonprogressive disease in PTMC [29], which was in contrast to our findings. In CPTMC, calcification was positively correlated with LNM, whereas in FPTMC and EnPTMC, no significant differences in LNM were found between tumors with or without calcification. Calcification in both subtypes was correlated with the unknown growth pattern but not with the occurrence of LNM. A cautious extent of surgery should be selected whether or not calcification is observed.

To investigate whether subtypes can be confirmed intraoperatively, differences in variant identification were compared between intraoperative frozen sections and postoperative paraffin sections. In intraoperative frozen sections, all the variants, except for the oncocytic variant, can be identified correctly according to the distinctive morphological characters, meaning that for almost all PTMCs, variants can be confirmed intraoperatively and thus used for the decision as to surgery extent.

There are three limitations in the present study. To clarify whether cases had LNM, we excluded the cases with no lymph node resected, which led to a slight rise in the rate of LNM. This issue was minimized by the rare occurrence of this situation. Also, the lower rate of LNM indicated that use of lymphadenectomy should be cautious in PTMC. The effect of surgery extent on data was another issue, being minimized by preoperative US of every patient. Because of color aberration, the oncocytic variant may be confused with other variants in frozen sections. As this variant does not have any prognostic implications in PTC [8] and was rare in PTMC, this limitation may not be important.

\section{Conclusion}

The present study establishes the three major variants in PTMC and their aggressive order: CPTMC $\gg$ FPTMC > EnPTMC. For reasons of distinct clinicopathological features and variant distribution, variant identification has important clinical significance in determination of lymphadenectomy, having an effect on individual treatments in PTMC. Patients with variantrelevant risk and other predictors, including male gender, age less than 45 years, tumor size, minimal ETE, calcification, and multifocality, are recommended to undergo prophylactic lymph node dissection.

Acknowledgments This work was partially supported by grants from National Natural Science Foundation of China (Grant Nos. 81402392, 81502322, 81472580, 81272282) and Tianjin Municipal Science and technology project (Grant No. 15JCQNJC12800).

\section{Compliance with ethical standards}

Conflict of interest The authors declare no conflict of interest. 
Open Access This article is distributed under the terms of the Creative Commons Attribution 4.0 International License (http:// creativecommons.org/licenses/by/4.0/), which permits unrestricted use, distribution, and reproduction in any medium, provided you give appropriate credit to the original author(s) and the source, provide a link to the Creative Commons license, and indicate if changes were made.

\section{References}

1. Davies L, Welch HG (2006) Increasing incidence of thyroid cancer in the United States, 1973-2002. JAMA 295(18):2164-2167. doi:10.1001/jama.295.18.2164

2. Lin JD (2010) Increased incidence of papillary thyroid microcarcinoma with decreased tumor size of thyroid cancer. Med Oncol (Northwood, London, England) 27(2):510-518. doi:10.1007/ s12032-009-9242-8

3. Chen AY, Jemal A, Ward EM (2009) Increasing incidence of differentiated thyroid cancer in the United States, 1988-2005. Cancer 115(16):3801-3807. doi:10.1002/cncr.24416

4. Jung CK, Little MP, Lubin JH et al (2014) The increase in thyroid cancer incidence during the last four decades is accompanied by a high frequency of BRAF mutations and a sharp increase in RAS mutations. J Clin Endocrinol Metab 99(2):E276-E285. doi:10.1210/jc.2013-2503

5. Pedrazzini L, Baroli A, Marzoli L et al (2013) Cancer recurrence in papillary thyroid microcarcinoma: a multivariate analysis on 231 patients with a 12-year follow-up. Minerva Endocrinol 38(3):269-279

6. Siddiqui S, White MG, Antic T et al (2016) Clinical and pathologic predictors of lymph node metastasis and recurrence in papillary thyroid microcarcinoma. Thyroid 26(6):807-815. doi:10.1089/thy.2015.0429

7. Soares P, Celestino R, Gaspar da Rocha A et al (2014) Papillary thyroid microcarcinoma: how to diagnose and manage this epidemic? Int J Surg Pathol 22(2):113-119. doi:10.1177/1066896913517394

8. Sak SD (2015) Variants of papillary thyroid carcinoma: multiple faces of a familiar tumor. Turk Patoloji Dergisi 31(Suppl 1):34 47. doi:10.5146/tjpath.2015.01313

9. Shi X, Liu R, Basolo F et al (2016) Differential clinicopathological risk and prognosis of major papillary thyroid cancer variants. J Clin Endocrinol Metab 101(1):264-274. doi:10.1210/ jc. $2015-2917$

10. Jun HH, Kim SM, Hong SW et al (2014) Warthin-like variant of papillary thyroid carcinoma: single institution experience. ANZ J Surg. doi:10.1111/ans.12725

11. Kazaure HS, Roman SA, Sosa JA (2012) Aggressive variants of papillary thyroid cancer: incidence, characteristics and predictors of survival among 43,738 patients. Ann Surg Oncol 19(6):18741880. doi:10.1245/s10434-011-2129-x

12. Moreno A, Rodriguez JM, Sola J et al (1996) Encapsulated papillary neoplasm of the thyroid: retrospective clinicopathological study with long term follow up. Eur J Surg Acta Chir 162(3):177-180

13. Lam AK, Lo CY, Lam KS (2005) Papillary carcinoma of thyroid: a 30-yr clinicopathological review of the histological variants. Endocr Pathol 16(4):323-330

14. Nikiforov YE, Erickson LA, Nikiforova MN et al (2001) Solid variant of papillary thyroid carcinoma: incidence, clinical-pathologic characteristics, molecular analysis, and biologic behavior. Am J Surg Pathol 25(12):1478-1484

15. Haugen BR, Alexander EK, Bible KC et al (2016) 2015 American Thyroid Association management guidelines for adult patients with thyroid nodules and differentiated thyroid cancer: The American Thyroid Association guidelines task force on thyroid nodules and differentiated thyroid cancer. Thyroid 26(1):1133. doi:10.1089/thy.2015.0020

16. Malandrino P, Russo M, Regalbuto C et al (2016) Outcome of the diffuse sclerosing variant of papillary thyroid cancer: a metaanalysis. Thyroid 26(9):1285-1292. doi:10.1089/thy.2016.0168

17. Liu LS, Liang J, Li JH et al (2016) The incidence and risk factors for central lymph node metastasis in $\mathrm{cN} 0$ papillary thyroid microcarcinoma: a meta-analysis. Eur Arch Oto-Rhino-Laryngol. doi: 10.1007/s00405-016-4302-0

18. Zhang L, Yang J, Sun Q et al (2016) Risk factors for lymph node metastasis in papillary thyroid microcarcinoma: older patients with fewer lymph node metastases. Eur J Surg Oncol 42(10):1478-1482. doi: 10.1016/j.ejso.2016.07.002

19. Thompson LD (2016) Ninety-four cases of encapsulated follicular variant of papillary thyroid carcinoma: a name change to Noninvasive Follicular Thyroid Neoplasm with Papillary-like Nuclear Features would help prevent overtreatment. Mod Pathol 29(7):698-707. doi:10.1038/modpathol.2016.65

20. Patel KN (2016) Noninvasive encapsulated follicular variant of papillary thyroid "cancer" (or not): time for a name change. JAMA Oncol 2(8):1005-1006. doi:10.1001/ jamaoncol.2016.0714

21. Rivera M, Tuttle RM, Patel S et al (2009) Encapsulated papillary thyroid carcinoma: a clinico-pathologic study of 106 cases with emphasis on its morphologic subtypes (histologic growth pattern). Thyroid 19(2):119-127. doi:10.1089/thy.2008.0303

22. Tunca F, Sormaz IC, Iscan Y et al (2015) Comparison of histopathological features and prognosis of classical and follicular variant papillary thyroid carcinoma. J Endocrinol Invest 38(12):1327-1334. doi:10.1007/s40618-015-0376-6

23. Lin HW, Bhattacharyya N (2010) Clinical behavior of follicular variant of papillary thyroid carcinoma: presentation and survival. Laryngoscope 120(Suppl 4):S163. doi:10.1002/lary.21627

24. Mao Y, Xing M (2016) Recent incidences and differential trends of thyroid cancer in the USA. Endocr Relat Cancer 23(4):313322. doi:10.1530/erc-15-0445

25. Sugitani I, Toda K, Yamada K et al (2010) Three distinctly different kinds of papillary thyroid microcarcinoma should be recognized: our treatment strategies and outcomes. World J Surg 34(6):1222-1231. doi:10.1007/s00268-009-0359-х

26. Miyauchi A (2016) Clinical trials of active surveillance of papillary microcarcinoma of the thyroid. World J Surg 40(3):516522. doi:10.1007/s00268-015-3392-y

27. Moon HJ, Kim EK, Chung WY et al (2011) Minimal extrathyroidal extension in patients with papillary thyroid microcarcinoma: is it a real prognostic factor? Ann Surg Oncol 18(7):1916-1923. doi:10.1245/s10434-011-1556-Z

28. Lu ZZ, Zhang Y, Wei SF et al (2015) Outcome of papillary thyroid microcarcinoma: study of 1,990 cases. Mol Clin Oncol 3(3):672-676. doi:10.3892/mco.2015.495

29. Fukuoka O, Sugitani I, Ebina A et al (2016) Natural history of asymptomatic papillary thyroid microcarcinoma: timedependent changes in calcification and vascularity during active surveillance. World J Surg 40(3):529-537. doi:10.1007/ s00268-015-3349-1 